This item was submitted to Loughborough's Research Repository by the author.

Items in Figshare are protected by copyright, with all rights reserved, unless otherwise indicated.

\title{
Theoretical and experimental study of a fluidic device as a fuel injector for
}

\section{natural gas engines}

PLEASE CITE THE PUBLISHED VERSION

PUBLISHER

(C) IMechE / Professional Engineering Publishing

VERSION

VoR (Version of Record)

LICENCE

CC BY-NC-ND 4.0

\section{REPOSITORY RECORD}

Chen, Rui, Q. Huang, and Gordon G. Lucas. 2009. "Theoretical and Experimental Study of a Fluidic Device as a Fuel Injector for Natural Gas Engines”. figshare. https://hdl.handle.net/2134/4497. 
This item was submitted to Loughborough's Institutional Repository (https://dspace.lboro.ac.uk/) by the author and is made available under the following Creative Commons Licence conditions.

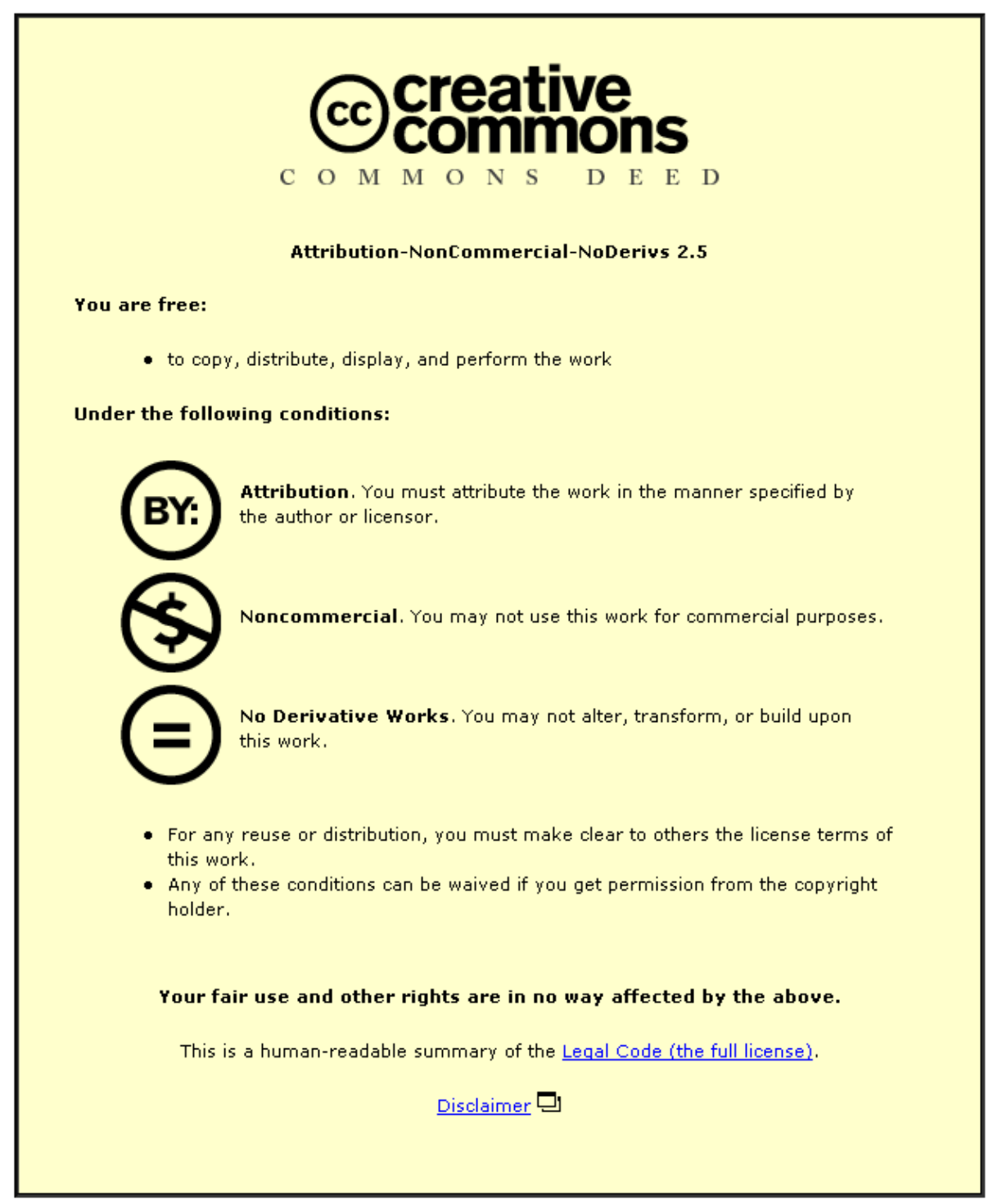

For the full text of this licence, please go to: http://creativecommons.org/licenses/by-nc-nd/2.5/ 


\title{
Theoretical and experimental study of a fluidic device as a fuel injector for natural gas engines
}

\author{
R Chen, Q Huang and G G Lucas \\ Department of Aeronautical and Automotive Engineering and Transport Studies, Loughborough University, Loughborough, \\ Leicestershire
}

\begin{abstract}
A novel fluidic gaseous fuel injector, designed for natural gas engines, has been tested and analysed in this paper. The injector is based on the use of a mono-stable fluidic amplifier. Its steady state and dynamic characteristics were tested and compared with several commercial solenoid gas injectors on a laboratory test rig. The results show that the fluidic gas injector is able to handle the large gas flowrates required by natural gas engines with a faster switching response and higher injection stability. Also, the steady state attachment and dynamic switching response of the jet flow in the fluidic amplifier were analysed by a mathematical simulation model. The agreement between predicted and experimental results is shown to be good.
\end{abstract}

Keywords: natural gas engine, gaseous fuel injection, fluidics, jet attachment, jet switching

\section{NOTATION}

a geometry constant

$b_{\mathrm{c}} \quad$ device control port width

$b_{\mathrm{s}} \quad$ device input nozzle width

$C_{\mathrm{d}} \quad$ discharge coefficient

$D \quad$ device offset

$F_{\mathrm{c}} \quad$ force from control port

$F_{\mathrm{s}} \quad$ force from jet flow

$J \quad$ momentum flux of jet flow

$J_{\mathrm{c}} \quad$ momentum flux of control flow

$J_{\text {main }}$ momentum flux of jet flow after being peeled

$J_{\text {peel }} \quad$ momentum flux of jet flow peeled by splitter

$J_{1} \quad$ momentum flux of output jet flow

$J_{2} \quad$ momentum flux of returned jet flow

$L_{\mathrm{sp}} \quad$ splitter distance

$m_{\mathrm{b}} \quad$ gas mass in the attachment bubble

$m_{\mathrm{c}} \quad$ gas mass in the control port

$N_{\mathrm{k}} \quad$ characteristic number

$p \quad$ pressure

$p_{\mathrm{b}} \quad$ bubble pressure

$p_{\mathrm{cp}} \quad$ pressure of the end of control port

$p_{\mathrm{o}} \quad$ ambient pressure

$p_{\mathrm{s}} \quad$ device supply pressure

$q \quad$ volume flowrate

$\bar{R} \quad$ gas constant

$\boldsymbol{R}$ radius vector

The MS was received on 29 April 1996 and was accepted for publication on 4 September 1997.
$R_{\mathrm{AL}} \quad$ average radius of attachment streamline

$R_{\mathrm{CL}} \quad$ average radius of jet flow centre-line

$s \quad$ jet flow axial distance from input nozzle

$s_{\mathrm{a}} \quad s$ of attachment point

$s_{\mathrm{o}} \quad s$ of the hypothetical origin of jet flow

$t$ time

$t_{\mathrm{b}} \quad$ response time of attachment bubble

$t_{\mathrm{c}} \quad$ response time of control port

$T$ temperature

$u \quad$ velocity

$w_{\mathrm{c}} \quad$ mass flowrate of control flow

$w_{\text {ent }}$ mass flowrate of one side jet flow entrainment

$w_{\mathrm{o}} \quad$ mass flowrate of jet flow at input nozzle

$w_{\text {ret }} \quad$ mass flowrate of returned jet flow

$w_{s_{2}} \quad$ mass flowrate of jet flow at $s_{\mathrm{a}}$

$w_{\text {vent }}$ mass flowrate of vent flow

$x_{\mathrm{a}}$

$y$

$y_{\mathrm{a}}$

$y_{\mathrm{sp}}$

Z

$\alpha \quad$ wall angle

$\beta \quad$ jet flow deflection angle

$\gamma \quad$ specific heats ratio [equations (17) to (19)]

$\gamma \quad$ angle made by attachment streamline extended with radius vector (Fig. 12)

$\delta \quad$ angle made by jet flow axis with radius vector

$\delta_{\mathrm{m}} \quad$ maximum possible value of $\delta\left(67^{\circ}\right)$

$\theta \quad$ angle made by jet flow axis extended with attachment wall 
$\begin{array}{ll}\rho & \text { density } \\ \sigma_{\mathrm{e}} & \text { jet flow spreading parameter }\end{array}$

\section{INTRODUCTION}

Natural gas consists primarily of methane. As an automotive fuel, natural gas can produce significantly less harmful exhaust emission. Also, since methane has a high knock resistance, having an octane rating of the order of 120 , natural gas engines can be operated at higher compression ratios. This results in improved fuel economies. Further, because the fuel is gaseous, there is no need for high fuelenrichment levels during cold-starts and transients. This has a significant effect on emissions and fuel economy.

It is envisaged that, to meet the increasingly stringent vehicle emission legislation, natural gas engines will have to employ a three-way catalyst in the exhaust system and be operated at the stoichiometric air-fuel ratio $(\mathbf{1}, \mathbf{2})$. This requires that the fuelling system must be capable of accurate control with fast response. A conventional gas fuelling system, such as venturi-type carburettors, cannot accurately compensate for the large changes in fuel density as a result of temperature and pressure changes without considerable complication. Hence, with a conventional gas fuelling system, three-way catalyst operation is difficult $(\mathbf{2}, \mathbf{3})$.

Gaseous fuel injection, combined with a sophisticated engine management system, is able to provide a good airfuel ratio control. However, since natural gas has a very low energy density, which is roughly one-third that of gasoline at $20 \mathrm{MPa}$ storage pressure, the conventional solenoid-type gas injector with normal injection pressure up to $1 \mathrm{MPa}$ must therefore be capable of handling a very large volume flowrate. Thus, not only will the injectors be large, with high pintle lifts, but also they will wear out rapidly because methane is not a good injector lubricant.

It is to meet these difficulties that the development of a novel fluidic gaseous fuel injector has been undertaken $(4,5)$. The fluidic gas injector has the potential of handling the large gas volume flowrates required by natural gas engines and providing a more accurate and faster response gas control than existing solenoid gaseous fuel injectors. Also, it can be arranged as a multipoint gas injection system in conjunction with the engine management system. A further advantage is that the amplification characteristic of the fluidic device can reduce the size and power requirement for the electrofluidic interface.

The purpose of this paper is to describe this fluidic gas injector and to investigate its capabilities. Also, a theoretical model was developed to analyse and simulate the steady state attachment and the dynamic switching process of the jet flow of the fluidic device. While not yet fully developed, the fluidic gas injector does show that it can overcome the shortcomings of both gas carburettors and solenoid-type gas injectors.

\section{FLUIDIC GASEOUS FUEL INJECTOR}

\subsection{Mono-stable fluidic amplifier}

The fluidic device being used in the fluidic gas injector is a mono-stable fluidic amplifier which is based on the wall reattachment Coanda effect. It has two control ports, namely the positive control port and the vacuum control port. These are located on each side of the input nozzle as shown in Fig. 1. The output flow channel which connects with the positive control port is called the stable output. The one that connects with the vacuum control port is called the unstable output.

If the pressure in the two control ports is atmospheric and the pressure of the gas stream entering the input nozzle is higher, the jet flow issuing through the input nozzle will be attached to the stable output. This is because the geometry design is such that this bias exists. When a gas flow at a sufficiently high pressure is initiated in the positive control port through an electrofluidic interface, the jet flow will be switched to the unstable output and remain there. When this gas flow is removed, the jet flow will be shifted back to reattach to the stable output. This switching method is called the 'positive control' method.

Alternatively, the electrofluidic interface can be used to close off the vacuum control port. Because of the jet flow entrainment, a 'vacuum' is created in the vacuum control port. This, too will cause the jet flow to be switched from the stable output to the unstable output till the vacuum control port is reopened to the atmosphere. This switching method is called the 'vacuum control' method.

Opening the electrofluidic interface and applying a sufficiently high pressure to the positive control port will result in a rapid switching of the jet flow from the stable output to the unstable output. Inversely, when the electrofluidic interface is closed, which cuts off the high pressure from the positive control port, the pressure in the control port will take some time to decay. The switching of the jet

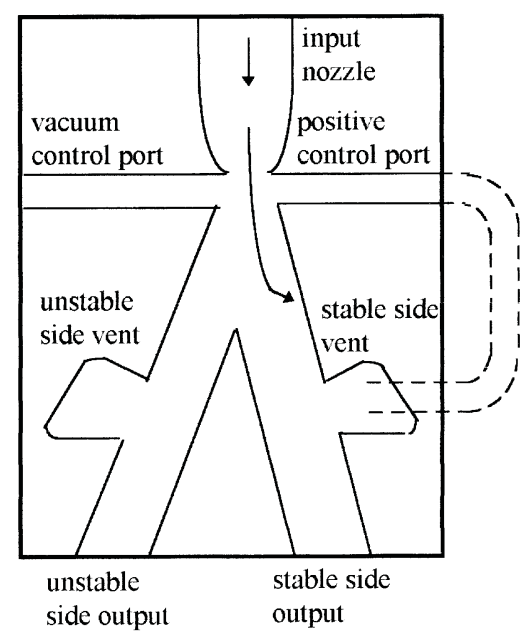

Fig. 1 Mono-stable fluidic device 
flow from the unstable output to the stable output will therefore be relatively slow.

The switching of the jet flow from the stable output to the unstable output is relatively slow by vacuum control, since it will take some time for the vacuum to be established. Inversely, when the vacuum control port is reopened suddenly, the jet flow will be switched from the unstable output to the stable output rapidly. This is because the necessary pressure change in the vacuum control port is relatively small and the switching is helped by the biased design.

Thus, the overall view is that in order to switch the jet flow from the stable output to the unstable output, the fast method is the positive control method. On the contrary, to switch the jet flow from the unstable output to the stable output, the fast method is the vacuum control method. However, there is an advantage in using the vacuum control method because the amplification (ratio of the supply pressure to the control pressure) is higher. Hence, the power requirement for the electrofluidic interface is lower.

The speed of switching the jet flow from the stable output to the unstable output by vacuum control can be largely improved by connecting the stable vent to the positive control port, as shown by the dashed lines in Fig. 1. This will create a slightly higher pressure than atmospheric in the positive control port when the jet flow is attached to the stable output. This pressure, in itself, is not high enough to cause the jet flow to be switched. However, it is a help when the vacuum control port is closed. This improvement has been proved by experiments in previous research (5) and was adopted in the fluidic gas injector.

\subsection{Fluidic gas injector}

In the fluidic gas injector, the fluidic device is arranged to be 'submerged' in an encapsulated gas reservoir. It is achieved by connecting the unstable vent and the unstable output to a gas reservoir as shown in Fig. 2. The stable output is connected to the engine inlet manifold through an isolating nozzle to provide gaseous fuel to the engine.

A conventional three-stage pressure regulator is used to reduce the gas pressure from $20 \mathrm{MPa}$ storage pressure to atmospheric. It is by connecting the output from the regulator to the reservoir that the 'submerged' pressure of atmospheric can be maintained.

The electrofluidic interface used in the injector is a standard central fuel injector (CFI) of Ford engines. Its driving system (type CS-453) operates in the specially designed 'peak and hold' mode for rapid opening and closing operations.

A low-pressure gas pump is connected to the gas reservoir and supplies a gas flow to the input nozzle of the fluidic device. When the electrofluidic interface is switched off, the vacuum control port is closed. The jet flow will be attached to the unstable output and returned to the reservoir. Under such conditions, the gas will simply be circulated around the pump, fluidic device and gas reservoir. The fluidic gas injector is switched off. When the

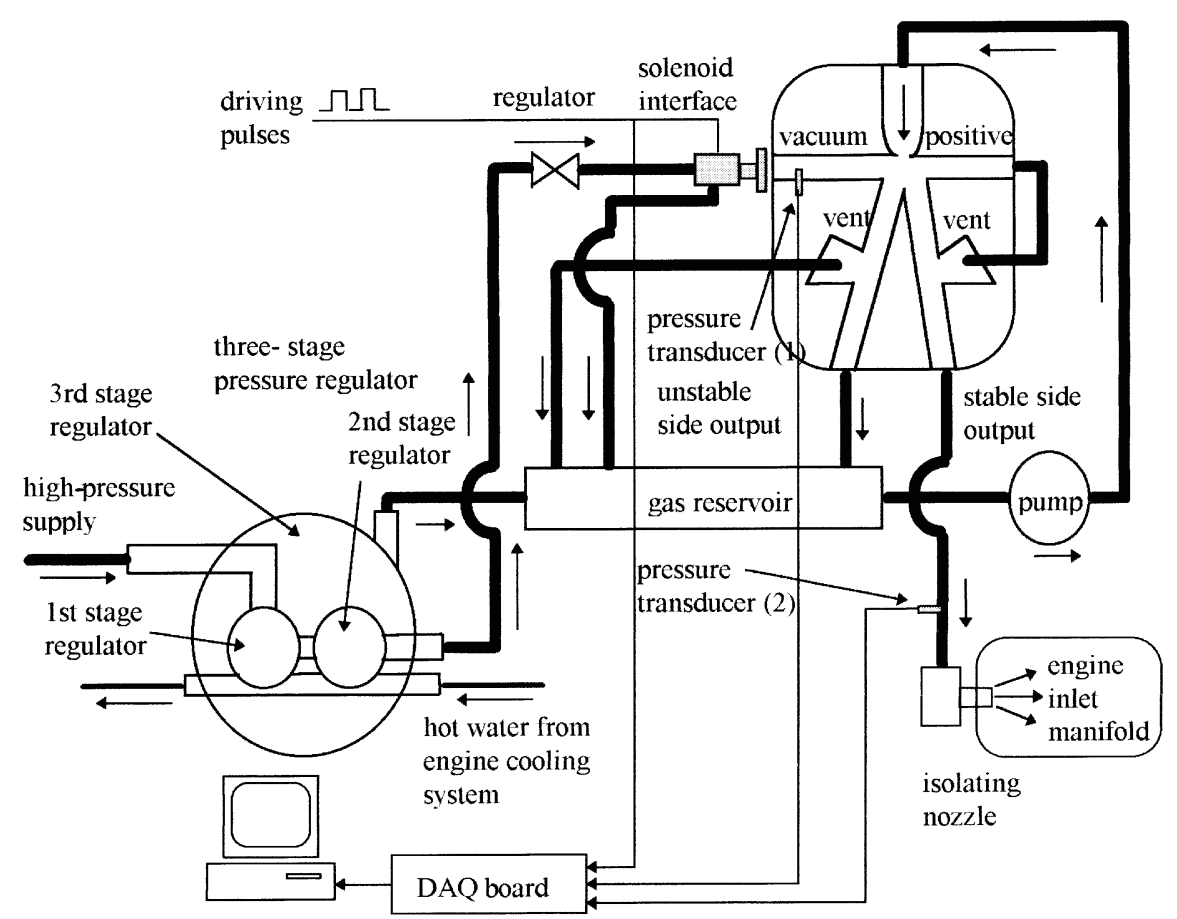

Fig. 2 A schematic set-up of the fluidic gaseous fuel injector and dynamic test rig 
interface is energized, the vacuum control port is opened. The jet flow will, then, be switched to the stable output and so provide fuel to the engine. The fluidic gas injector is switched on.

\section{EXPERIMENTAL STUDY}

The experimental work was focused on the steady state flow deliveries of two versions of the fluidic device and the dynamic switching characteristic together with cycle-tocycle variability of a prototype of the fluidic gas injector. Several commercial solenoid-type gas injectors were also tested and the results were compared with those from the prototype.

\subsection{Steady state test on fluidic devices}

The potential of flow-handling capability of fluidic devices was estimated by steady state flow tests operating on air under varying device supply pressure and output restricting nozzle size. Two versions of fluidic device, a small version with an input nozzle of $1 \mathrm{~mm}$ by $2 \mathrm{~mm}$ and a large version with an input nozzle of $1.5 \mathrm{~mm}$ by $3 \mathrm{~mm}$, were tested. Figure 3 shows the comparison of the results between commercial Servojet gas injectors and fluidic devices. The Servojet data were based on natural gas injection and provided by the manufacturer. The dynamic flow deliveries of fluidic devices were calculated from the results of steady state tests with compressed air.

Three Servojet units were used, namely SP-014, SP-021 and SP-051. The supply pressure for these injectors was $700 \mathrm{kPa}$. It can be seen that the dynamic flow delivery by the large version of fluidic device at a supply pressure of $150 \mathrm{kPa}$ and output restriction nozzle of $3 \mathrm{~mm}$ in diameter is the same as that of the SP-051, which is the smallest Servojet unit. The small version fluidic device at the same condition is about 40 per cent lower. In order to achieve the

Fluidic injector: $P S=150 \mathrm{kPa}$, Servojet injector: $P S=700 \mathrm{kPa}$

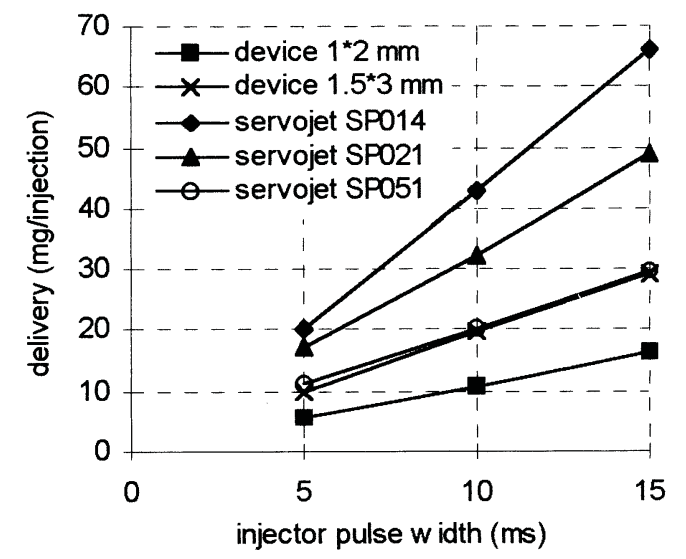

Fig. 3 Comparison of the dynamic flow delivery between fluidic devices and Servojet injectors flow delivery of the SP-014, which is approximately twice as much as that of the SP-051, two large version fluidic devices can be stacked together for larger engine applications. The advantages of a stacked arrangement are the flexibility of adapting the fluidic unit for various engine sizes and the avoidance of a slow response time.

\subsection{Dynamic test on the fluidic gas injector}

The large version of fluidic device was used in the prototype of fluidic gas injector due to its higher flow delivery ability. In order to evaluate dynamic switching characteristics of the fluidic gas injector and to compare them with those from commercial gas injectors, a laboratory test rig was built as shown in Fig. 2. Two fastresponding Entran pressure transducers (type EPI-M4) were used. One was fitted on the vacuum control port to detect the response of the solenoid interface, i.e. the control flow signal. The other one was fitted on the stable side output of the fluidic device to monitor the response of the fluidic gas injector.

A PC-based data acquisition (DAQ) system was developed to collect test results and to analyse switching response times and cycle-to-cycle variations. The software used in the PC was written in 'LabVIEW', a language developed by National Instrument. An Amplicon DAQ board was chosen for its high frequency and low cost. Advanced linking driver software had to be developed in order to operate the Amplicon board in the LabVIEW language satisfactorily.

Figure 4 shows the switching response times of the fluidic gas injector measured by pressure transducer (2) in Fig. 2. It is the response time between the input driving signal to the solenoid interface and the output pressure signal of the fluidic injector. Figure 5 shows the switching cycle-to-cycle variations. The supply pressure of the injector was $150 \mathrm{kPa}$. Each data point in the figures was obtained from 500 consecutive cycles.

It can be seen that as the control pressure increases, the

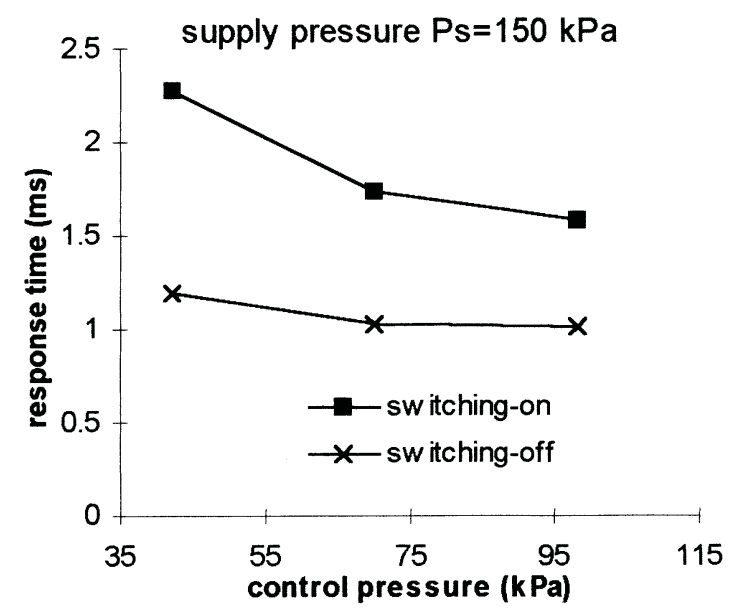

Fig. 4 Switching response times of the fluidic gas injector (by vacuum control) 


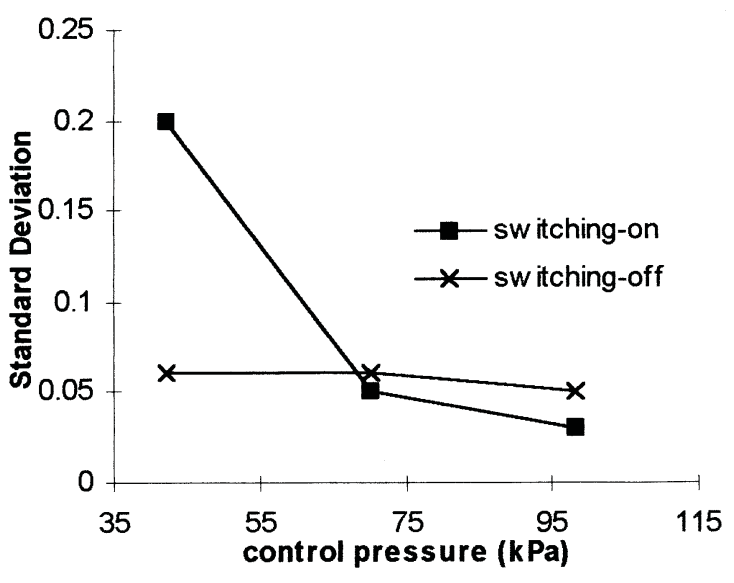

Fig. 5 Switching cycle-to-cycle variations of the fluidic gas injector (by vacuum control)

switching-on response time and cycle-to-cycle variation which is measured by the standard deviation (SD) decreases. This is because, when the fluidic gas injector is switching-on by using the vacuum control method, the jet flow is switched from the unstable output to the stable output. The switching is mainly due to the mono-stable nature of the device. A higher control pressure will accelerate the switching and, therefore, reduce the response time and increase the switching stability.

On the other hand, the switching-off response time and cycle-to-cycle variation do not change very much as the control pressure increases. This is because the process by which the jet flow is switched back to the unstable output from the stable output is a result of the pressure difference across the jet flow. This difference is not affected by the variation of control pressure since the control port is closed.

It can be seen that at $100 \mathrm{kPa}$ control pressure and $150 \mathrm{kPa}$ supply pressure the switching-on response time and the cycle-to-cycle variation of the fluidic gas injector is $1.6 \mathrm{~ms}$, with an SD of 0.03 . The switching-off response time and the cycle-to-cycle variation is $1 \mathrm{~ms}$ with an SD of 0.06 .

\subsection{Dynamic tests on commercial gas injectors}

The commercial gas injectors available for comparison in this research were three Servojet gas injectors and one Bosch gas injector. Since no dynamic characteristics were available for these proprietary injectors, it was decided to carry out similar dynamic tests on the laboratory test rig, so that their switching performances could be compared with those from the fluidic injector system.

Figures 6 and 7 show the switching-on and -off response times of these injectors under varying supply pressures. It is known that the supply pressures of these injectors on gas engines are in the range of $700-1000 \mathrm{kPa}$. However, the maximum pressure used in the test was $350 \mathrm{kPa}$ due to limited peak pressure availability in the laboratory. It can be seen that the switching-on response time increases as

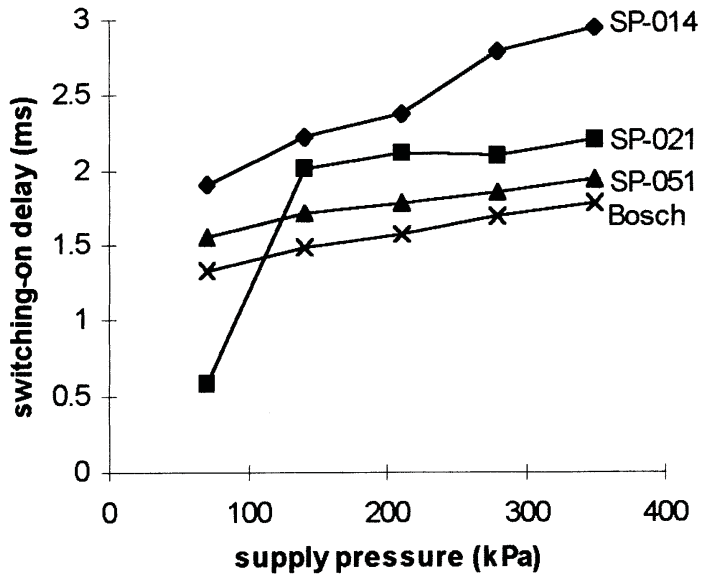

Fig. 6 Switching-on response times of commercial gas injectors

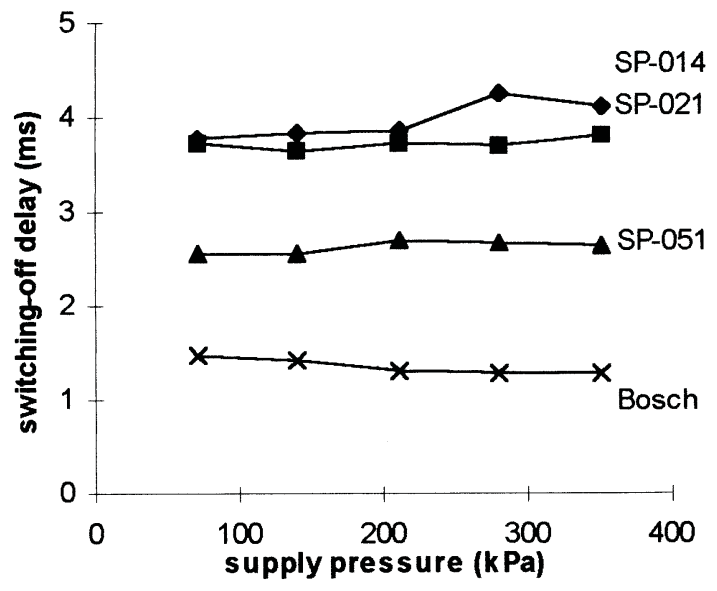

Fig. 7 Switching-off response times of commercial gas injectors

the supply pressure increases for all four injectors. As mentioned earlier, the switching-on time achieved by the fluidic gas injector at $100 \mathrm{kPa}$ control pressure and $150 \mathrm{kPa}$ supply pressure is in the region of $1.6 \mathrm{~ms}$. Therefore, it is reasonable to predict that the fluidic gas injector has a faster switching speed than all four proprietary injectors, when operated at their normal pressure.

For the switching-off response the Bosch unit showed the best response time $(1.4 \mathrm{~ms})$ among the commercial injectors. However, the switching-off response time achieved by the fluidic gas injector at $150 \mathrm{kPa}$ supply pressure and $100 \mathrm{kPa}$ control pressure is $1 \mathrm{~ms}$. It should be noted that the Bosch gas injector used here is a relatively small unit designed for gas engines possibly with a capacity below 2 litres. Therefore, it has the advantage of a light armature and a small passage area, resulting in a faster response than the Servojet injectors.

Figures 8 and 9 show the cycle-to-cycle variations of these commercial injectors. It can be seen that the Bosch unit showed extremely consistent cycle-to-cycle variations with an SD of 0.02 for both switching on and off. This is largely due to its compactness and lightweight design. In 


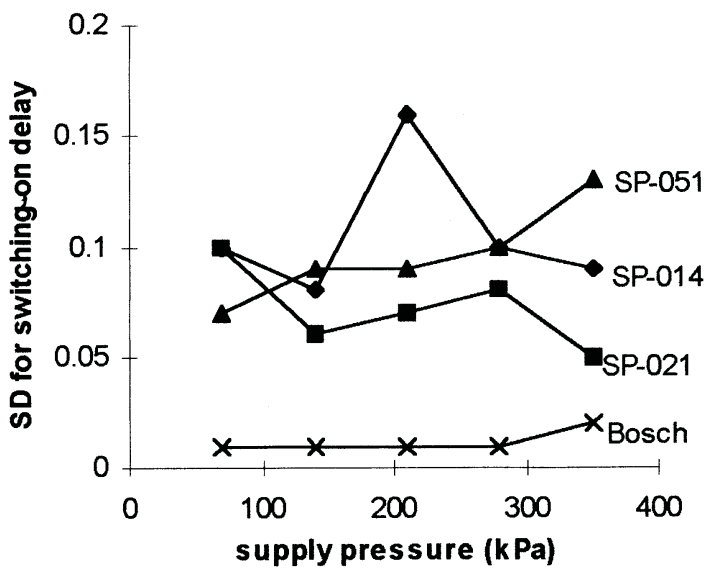

Fig. 8 Switching-on cycle-to-cycle variations of commercial gas injectors

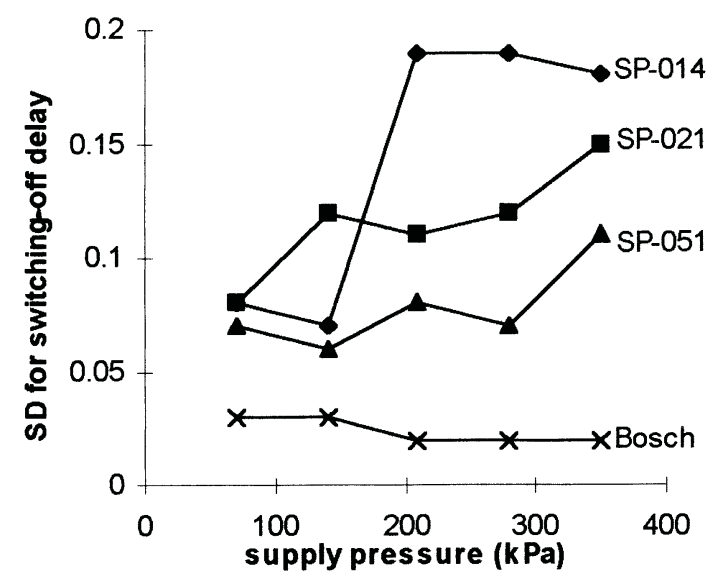

Fig. 9 Switching-off cycle-to-cycle variations of commercial gas injectors

comparison, the switching cycle-to-cycle variations of the fluidic gas injector are with an SD of 0.03 for switching on and an SD of 0.06 for switching off at $150 \mathrm{kPa}$ supply pressure and $100 \mathrm{kPa}$ control pressure.

\section{ANALYTICAL MODEL}

The response time of the fluidic gas injector obtained in the tests can be divided into two parts. One is the response time of the electrofluidic interface. The other one is the response time of the fluidic device. To achieve an overall fast response, the response speed of the interface may be directly improved by introducing a dedicated design. However, the improvement in switching response of the fluidic device is somewhat uncertain since the fluidic device geometry design is largely based on a 'trial-and-error' approach. In this section, an analytical model is produced in an attempt to analyse the mechanism of the switching process and to identify some critical parameters responsible for the speed of switching.

\subsection{Steady state attachment theory}

When a fluidic device is turned on, a jet flow is exited from the input nozzle. It entrains the surrounding fluid from both sides. Because of the presence of device walls, some of the molecules of the fluid between the jet flow and the wall will be evacuated. This results in a low-pressure region on each side of the jet flow into which there is a counter-flow down the wall. When the pressures of these two regions lose balance, the jet flow will bend towards the lower pressure region and form an enclosed lower pressure bubble. The jet flow will then impact with the wall and flow along it. This phenomenon, illustrated in Fig. 10, is called the jet flow attachment. The lower pressure bubble is called the attachment bubble.

\subsubsection{Attachment assumptions}

To analyse the jet flow attachment, a few assumptions are needed:

1. The fluidic device is assumed to be two dimensional.

2. The jet flow is assumed to be a free jet having the Goertler velocity profile:

$$
u=\left[\frac{3 J \sigma_{\mathrm{e}}}{4 \rho\left(s+s_{\mathrm{o}}\right)}\right]^{1 / 2} \operatorname{sech}^{2} \frac{\sigma_{\mathrm{e}} y}{s+s_{\mathrm{o}}}
$$

3. The momentum flux peeled off by the splitter is assumed to be determined by the relative position of the assumed velocity profile with the splitter (6) as shown in Fig. 11. Therefore, the peeled-off momentum flux by the splitter is

$$
J_{\text {peel }}=\int_{-\infty}^{-y_{\mathrm{sp}}} \rho u^{2} \mathrm{~d} y
$$

and the rest of the jet flow momentum flux then becomes

$$
J_{\text {main }}=\int_{-y_{\mathrm{sp}}}^{\infty} \rho u^{2} \mathrm{~d} y
$$

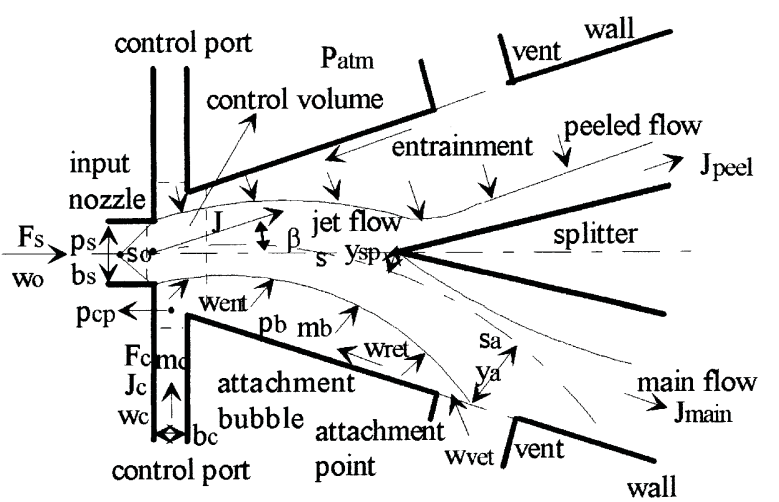

Fig. 10 Jet flow attachment of the fluidic device 


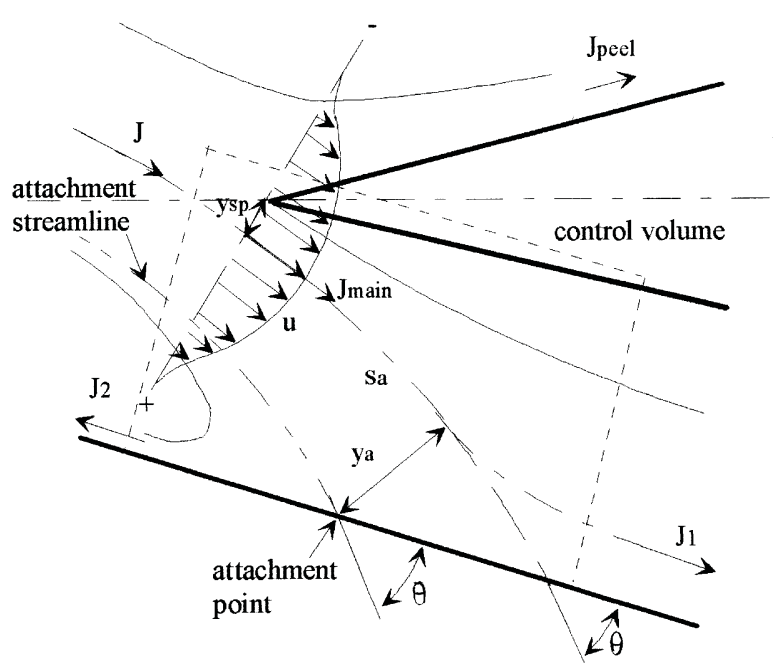

Fig. 11 Jet flow attachment point

4. The momentum flux is assumed to be conserved in the control volume surrounding the attachment point (Fig. 11).

5. The average angle of the momentum flux of the jet flow is assumed to be essentially the same as the angle made by the centre-line extended with the attachment wall $\theta$. Therefore,

$$
J_{\text {main }} \cos \theta=J_{1}-J_{2}
$$

where $J_{2}$ is the momentum flux of the jet flow returned into the attachment bubble and can be expressed as

$$
J_{2}=\int_{y_{\mathrm{a}}}^{\infty} \rho u^{2} \mathrm{~d} y
$$

and $J_{1}$ is the rest of the main momentum flux of the jet flow

$$
J_{1}=\int_{-y_{\mathrm{sp}}}^{y_{\mathrm{a}}} \rho u^{2} \mathrm{~d} y
$$

Substituting equations (2), (3) and (5), (6) into equation (4) provides

$$
\left(t_{\mathrm{sp}}-\frac{t_{\mathrm{sp}}^{3}}{3}+\frac{2}{3}\right) \cos \theta=2\left(t_{\mathrm{a}}-\frac{t_{\mathrm{a}}^{3}}{3}\right)+t_{\mathrm{sp}}-\frac{t_{\mathrm{sp}}^{3}}{3}-\frac{2}{3}
$$

where

$$
t_{\mathrm{a}}=\tanh \frac{\sigma_{\mathrm{e}} y_{\mathrm{a}}}{s_{\mathrm{a}}+s_{\mathrm{o}}}
$$

$$
t_{\mathrm{sp}}=\tanh \frac{\sigma_{\mathrm{e}} y_{\mathrm{sp}}}{s_{\mathrm{sp}}+s_{\mathrm{o}}}
$$

If the splitter is far enough from the jet exit nozzle, $y_{\mathrm{sp}}$ can be considered to be infinite and $t_{\mathrm{sp}}=1$. Equation (7) then becomes

$$
\cos \theta=\frac{3}{2} t_{\mathrm{a}}-\frac{1}{2} t_{\mathrm{a}}^{3}
$$

This is a preliminary result of equation (4) without considering the effect of the splitter (7).

6. The attachment streamline of the jet flow is assumed to be a sinusoidal function, as shown in Fig. 12. This assumption is based on the fact that the pressure inside the bubble is not constant and the attachment distance becomes infinite as the wall angle of the inclination approaches $67^{\circ}(\mathbf{8})$. The jet attachment streamline path function can then be expressed as

$$
\boldsymbol{R}=a \sin \left(\frac{\pi}{2} \frac{\delta}{\delta_{\mathrm{m}}}\right)
$$

\subsubsection{Equilibrium state of the attachment bubble}

To maintain a stable attachment of the jet flow, there must be an equilibrium state of mass flowrates across the attachment bubble. In the steady state analysis, the attachment side control port is assumed to be blocked. The only flow into the attachment bubble is the returned jet flow. Therefore,

$$
w_{\mathrm{ent}}=w_{\mathrm{ret}}
$$

where the jet flow entrained mass flowrate from one side of the jet flow $w_{\text {ent }}$ can be obtained as

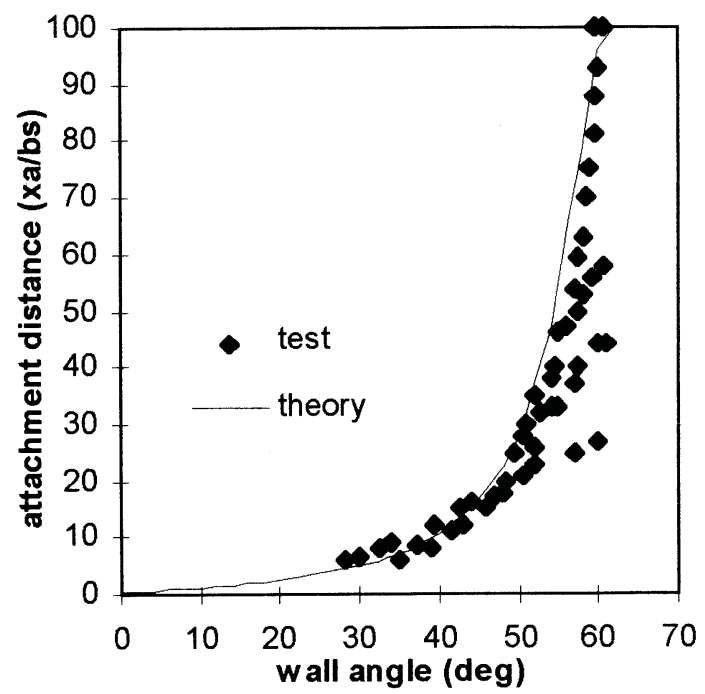

Fig. 12 Jet flow steady state attachment distances 


$$
w_{\mathrm{ent}}=\frac{w_{s_{\mathrm{a}}}-w_{\mathrm{o}}}{2}
$$

where

$$
\begin{aligned}
& w_{s_{\mathrm{a}}}=\left.\int_{-\infty}^{\infty} \rho u \mathrm{~d} y\right|_{s=s_{\mathrm{a}}} \\
& w_{\mathrm{o}}=\left.\int_{-\infty}^{\infty} \rho u \mathrm{~d} y\right|_{s=0}
\end{aligned}
$$

The returned mass flowrate $w_{\text {ret }}$ can be obtained as

$$
w_{\text {ret }}=\left.\int_{\infty}^{y_{\mathrm{a}}} \rho u \mathrm{~d} y\right|_{s=s_{\mathrm{a}}}
$$

Since gas is the fluid medium, its compressibility has to be considered when the jet is issued from the device input nozzle. For simplicity, in this research, the mass flowrate of the jet flow is calculated from the one-dimensional orifice compressible flowrate equation. When the ratio of the ambient pressure $p_{\mathrm{o}}$ to the device input nozzle supply pressure $p_{\mathrm{s}}$ is larger than the criterion

$$
\left(\frac{p_{\mathrm{o}}}{p_{\mathrm{s}}}\right)_{\mathrm{chok}}=\left(\frac{2}{\gamma+1}\right)^{\gamma /(\gamma-1)}
$$

the jet mass flowrate is

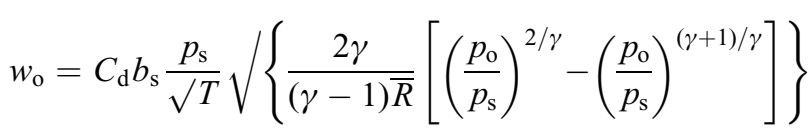

When the pressure ratio is lower than the critical ratio, the jet flow is choked and the mass flowrate should not be affected by the downstream pressure. The mass flowrate becomes

$$
w_{\mathrm{o}, \mathrm{chok}}=C_{\mathrm{d}} b_{\mathrm{s}} \frac{p_{\mathrm{s}}}{\sqrt{ } T} /\left[\frac{\gamma}{\boldsymbol{R}}\left(\frac{2}{\gamma+1}\right)^{(\gamma+1) /(\gamma-1)}\right]
$$

Solving equations (1), (7) to (9) and (11) to (19), together with the relevant geometric relations as shown in Fig. 13 simultaneously, all the steady state jet flow attachment information, such as the attachment distance perpendicular to the jet flow axis $y_{\mathrm{a}}$, the attachment distance along the attaching wall $x_{\mathrm{a}}$, the jet flow attachment angle $\theta$, the attachment bubble volume, etc., can be obtained.

For steady state attachment experimental study, much has been achieved by previous researchers, particularly by Foster and Parker (9). Therefore, the results of the steady state attachment theory can be compared directly with these well-established experimental results. Figure 12 shows the comparison in which the test data were chosen

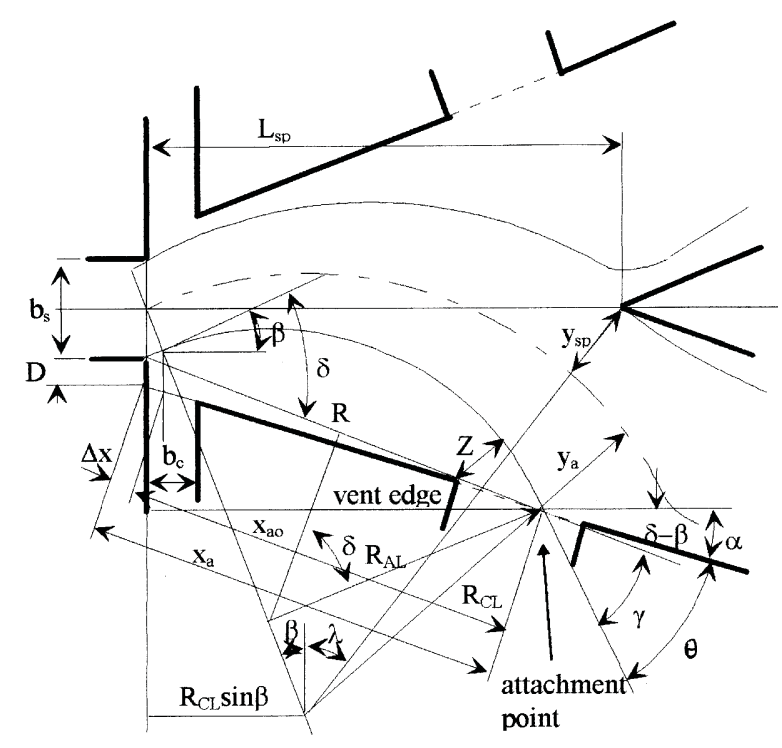

Fig. 13 Geometric relations of the jet flow attachment

from Bourque and Newman (7) and the theoretical steady state attachment distances $x_{\mathrm{a}}$ were calculated from the steady state attachment theory. It can be seen that the calculated results agree well with the test data. The attachment distance increases as the wall angle increases. As the wall angle approaches the limitation value of $67^{\circ}$, the attachment distance approaches infinity.

\subsection{Jet flow dynamic switching model}

The switching response time of a fluidic device is the time taken for the jet flow to be switched to the opposite wall after the control flow is applied. In this section, this response time was calculated using a jet flow dynamic switching model based on the use of a quasi-steady assumption. The model consists of two parts, the jet flow dynamics and the control port dynamics.

\subsubsection{Jet flow dynamics}

In a mono-stable fluidic device, the jet flow is initially attached to the stable output. If a control flow is applied on the positive control port, the jet flow will be deflected by the pushing force of the control flow. This deflection can be represented by a deflection angle $\beta$. It can be obtained by balancing the vertical force with the control volume outside the device input nozzle, as shown in Fig. 10:

$$
\tan \beta=\frac{F_{\mathrm{c}}}{F_{\mathrm{s}}}
$$

in which the force from the control port is

$$
F_{\mathrm{c}}=J_{\mathrm{c}}+b_{\mathrm{c}}\left(p_{\mathrm{cp}}-p_{\mathrm{b}}\right)
$$

and the force from the jet flow is 


$$
F_{\mathrm{s}}=J+b_{\mathrm{s}} p_{\mathrm{s}}
$$

When the control flow is introduced to the attachment bubble, the equilibrium state of mass flowrates across the attachment bubble will be broken. The newly introduced control flow will not only change the mass contained in the bubble $m_{\mathrm{b}}$ but also the flows crossing the bubble, until a new mass flowrate balance across the attachment bubble can be reached:

$$
\frac{\mathrm{d} m_{\mathrm{b}}}{\mathrm{d} t}+w_{\mathrm{ret}}+w_{\mathrm{c}}=w_{\mathrm{ent}}
$$

Rearranging this equation, the dynamic response time of the bubble mass change due to the addition of the control flow can be obtained as

$$
\mathrm{d} t_{\mathrm{b}}=\frac{\mathrm{d} m_{\mathrm{b}}}{w_{\text {ent }}-w_{\text {ret }}-w_{\mathrm{c}}}
$$

The dynamic response time of the attachment bubble mass change can be obtained by using the quasi-steady assumption, i.e. assuming that the mass flowrate of the control flow is increased step by step. For each step, the mass flowrates across the attachment bubble can be assumed to reach a new balance [equation (23)]. The bubble mass can be obtained from the bubble volume by assuming the perfect gas. Therefore, by comparing the new bubble mass with the previous step, the bubble response time due to the step control mass flowrate increase can be obtained by equation (24).

As the control flow increases, the attachment bubble expands and the attachment point moves away from the input nozzle. When the attachment point passes the vent edge, an additional flow will be introduced into the attachment bubble through the 'opening' vent, as shown in Fig. 10. As a result, the equilibrium of the mass flows across the attachment bubble becomes

$$
\frac{\mathrm{d} m_{\mathrm{b}}}{\mathrm{d} t}+w_{\mathrm{ret}}+w_{\mathrm{c}}+w_{\mathrm{vent}}=w_{\mathrm{ent}}
$$

in which the mass flowrate of vent flow $w_{\text {vent }}$ is calculated from the assumption of incompressible flow due to the low pressure drop:

$$
w_{\mathrm{vent}}=Z C_{\mathrm{d}} \sqrt{ }\left[2 \rho\left(p_{\mathrm{atm}}-p_{\mathrm{b}}\right)\right]
$$

So far, if the equations associated with the addition of control flow are solved together with steady state jet flow attachment theory by the quasi-steady assumption simultaneously, ideally the response time of switching the jet flow off the attachment wall can be obtained by summing the elementary response times shown in equations (25) and (26) until the attachment point moves off the wall.

However, in practice, it is inevitable that there is a nominal length of the control port between the output of the electrofluidic interface and the entrance point into the attachment bubble. There will be a response delay time between the point when the control flow is available at the electrofluidic interface and the point when the control flow starts to affect the jet flow due to the existence of the 'dead volume' of the control port. This part response delay seriously affects the jet flow switching response as well.

\subsubsection{Control port dynamics}

From equation (20), it can be seen that the pressure of the end of the control port $p_{\text {cp }}$ plays an important part in jet flow deflection. This pressure and the response delay due to the 'dead volume' of the control port can be analysed by the method of characteristics, by changing the partial derivative of the momentum and the continuity equations to total derivatives under certain conditions (10):

$$
\begin{aligned}
& N_{\mathrm{k}} \frac{\mathrm{d} q^{\prime}}{\mathrm{d} t^{\prime}} \pm \frac{\mathrm{d} p^{\prime}}{\mathrm{d} t^{\prime}}+q^{\prime}=0 \\
& \frac{\mathrm{d} x^{\prime}}{\mathrm{d} t^{\prime}}= \pm 1
\end{aligned}
$$

where a prime denotes normalization. The control flow from the solenoid interface can be obtained from equations (17) to (19), in which the gas flow through the solenoid orifice is assumed to be a one-dimensional compressible flow. The size of the orifice was established from steady state flow tests using a liquid fuel.

Because of the existence of the 'dead volume' of the control port, it will take some time to build up the pressure in the control port after the control flow is available in the electrofluidic interface. By using the quasi-steady method and the dynamic mass flow equilibrium with the control volume of the control port,

$$
\frac{\mathrm{d} m_{\mathrm{c}}}{\mathrm{d} t}=w_{\mathrm{c}}
$$

the dynamic response time to raise the control port pressure can be obtained:

$$
\mathrm{d} t_{\mathrm{c}}=\frac{\mathrm{d} m_{\mathrm{c}}}{w_{\mathrm{c}}}
$$

where the mass of the gas in the control port $m_{\mathrm{c}}$ is obtained from the control port pressure obtained from equations (27a) and (27b) by assuming the perfect gas. Therefore, the overall control port response can be obtained by summing the elementary response time obtained from equation (29).

Finally, the total response time of the fluidic device to the control flow (apart from the solenoid interface delay to the driving signal) can be obtained by summing the overall control port delay and the jet flow switching delay.

Proc Instn Mech Engrs Vol 212 Part D 


\subsubsection{Model validation}

The model being discussed so far can simulate the jet flow switching off the initial attached wall by the positive control method (i.e. the jet flow being switched from the stable side output to the unstable side output by applying a sufficient control flow to the positive control port). Therefore, to evaluate the accuracy of the model, the experiment has to be carried out with the positive control method.

Figure 14 shows the validation test rig in which the fluidic device was switched by the positive control method. The small version fluidic device was chosen. This is due to the fact that the solenoid interface available for the test is only able to provide a sufficient control flowrate for the small version device when the positive control method is being used.

Figure 15 shows a typical comparison between experimental and theoretical results. Three groups of measured pressure data, namely control port pressure, stable output pressure and unstable output pressure, together with the driving signal, are shown. The time-scale 'zero' indicates the point where the solenoid opens. It can be seen that the solenoid takes $0.7 \mathrm{~ms}$ to open after the driving pulse is applied. Before the control port pressure reaches its maximum value, the jet flow attachment on the stable output will start to break down (as the stable output pressure starts to fall down in the plot). Meanwhile, the unstable output

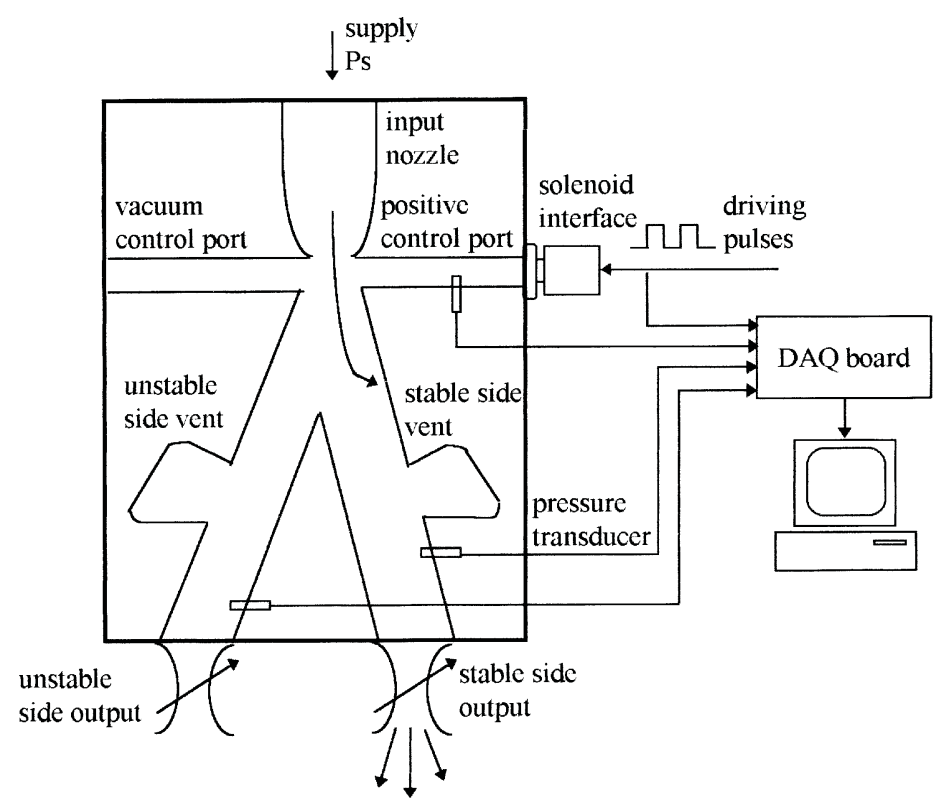

Fig. 14 Fluidic device dynamic test rig by the positive control method

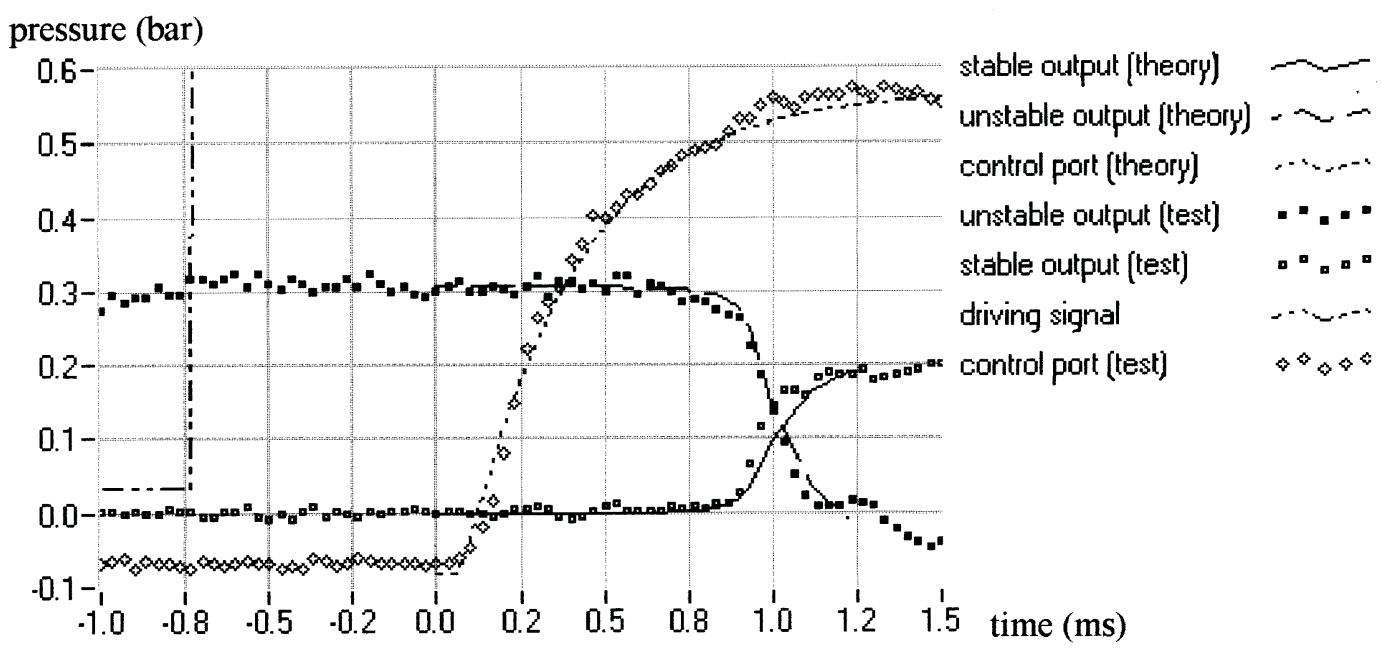

Fig. 15 Jet flow reattachment switching response 
pressure increases, which indicates that the jet flow has reached the opposite wall, i.e. unstable output.

It can be seen that the simulated results from the modelling agree well with the experimental ones. The calculated results for the control port pressure were obtained from the 'method of characteristics' and the two output pressure traces were obtained from the 'dynamic attachment model'. It should be pointed out that some input parameters of initial conditions are required for the model. They are the geometry of the device, supply pressure to the device input nozzle $p_{\mathrm{s}}$, control pressure to the solenoid interface $p_{\mathrm{c}}$, initial pressure inside the attachment bubble $p_{\mathrm{b}}$ (tested), maximum pressure in the control port (tested), the characteristic number $N_{\mathrm{k}}$ and the spreading parameter $\sigma_{\mathrm{e}}$.

One important parameter that has a significant influence on the simulated results is the spreading parameter $\sigma_{\mathrm{e}}$. It is worth noting that the $\sigma_{\mathrm{e}}$ value used in the modelling for the results shown in Fig. 15 is 35.5. This is much higher than the value of 7.5 chosen for the Goertler free jet flow assumption.

This discrepancy can be explained as follows. When the Goertler equation is introduced, the jet flow is assumed to be two dimensional, i.e. the jet flow entrainment, attachment and switching are independent from the depth of the device. However, the device used for the tests has a limited depth with an aspect ratio (ratio of device depth to input nozzle width) of 2 . The jet flow entrainment has been largely reduced due to the reduced contact area between the jet flow and the surrounding fluid. Therefore, by increasing the value of the jet flow spreading parameter $\sigma_{\mathrm{e}}$, which is inversely proportional to the jet flow entrainment, the model can be somewhat 'tuned' so that realistic results can be predicted. The relationship between the jet flow spreading parameter and the device aspect ratio has also been discussed in Epstein's work (11).

Figure 16 shows the tested and the predicted results of the jet flow switching response delays when the solenoid interface is energized by the positive control method at $150 \mathrm{kPa}$ supply pressure. The test data was an average of

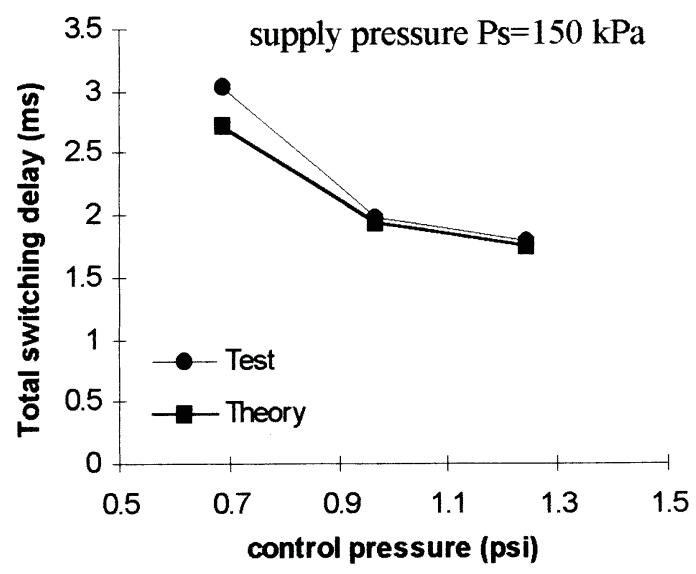

Fig. 16 Switching-on response of fluidic devices by positive control
500 consecutive cycles. The inputs and initial conditions for the model are the same as discussed in Fig. 15, but with a change in the interface control pressure. Clearly, the model is able to predict the fluidic device switching response delay time by the positive control method with an error of 1-8 per cent compared to the test results.

Figure 17 shows one of the predictions obtained from the dynamic reattachment model. It can be seen that the jet flow switching response time is almost linearly proportional to the control port length. This suggests that the increase in the 'dead volume' in the control port due to the increase in length will considerably reduce the jet flow switching speed. It is therefore important to fit the electrofluidic interface as close as possible to the fluidic device.

\section{FUTURE WORK}

1. To improve the packaging technique for the fluidic device and electrofluidic interface so that the control port 'dead volume' can be minimized.

2. To develop a dedicated electrofluidic interface for the fluidic gas injector.

3. To improve and modify the dynamic switching model to predict the switching response speed by the vacuum control method.

4. To implement the prototype of a fluidic gas injector on a research gas engine so that the engine operating characteristics by the fluidic gas injector can be compared with those of solenoid gas injectors and basic gas carburettors.

\section{CONCLUSION}

1. A novel gaseous fuel injector based on the use of a mono-stable fluidic device was proposed. Its working principle was described and verified by experiments.

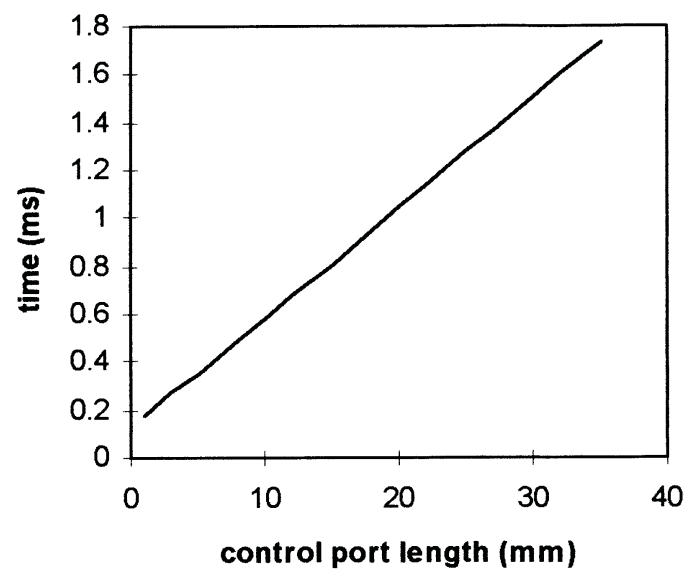

Fig. 17 Switching response of fluidic devices affected by the control port length 
2. The proposed fluidic gas injector is able to handle the large gas flowrate required by natural gas engines. The larger flowrate can be obtained by stacking the multilayer fluidic devices.

3. It was demonstrated that the fluidic gas injector is able to achieve faster switching response times than commercial solenoid gas injectors with a typical switching-on response time of $1.6 \mathrm{~ms}$ and switching-off response time of $1 \mathrm{~ms}$. Also, the fluidic gas injector has shown a good switching cycle-to-cycle stability with standard deviations of 0.03 and 0.06 for switching on and off respectively.

4. An analytical model was established to predict the jet flow switching response in a fluidic device. The predicted results from the model agreed well with experimental results with an error within 8 per cent. However, the model can only be used to predict the jet flow switching from the stable side output to the unstable side output with a positive control method. Further modifications are required for wider applications.

\section{ACKNOWLEDGEMENTS}

The financial support from British Gas plc for this project is gratefully acknowledged. The authors also wish to record their appreciation of technical assistance provided by $\mathrm{Mr}$ Alan Brightwell and Ms Rachel Palmer, British Gas plc, Mr John Elliott, Preci-Spark Limited UK, and Technician Mr Derek Harkis, Loughborough University.

\section{REFERENCES}

1 Klimstra, J., et al. Catalytic converters for natural gas fuelled engines - a measurement and control problem. SAE paper $872165,1987$.

2 Klimstra, J., et al. Carburettors for gaseous fuel-on air-fuel ratio homogeneity and flow restriction. SAE paper 892141, 1989.

3 Pereault, M., Allard, G., et al. An alternative to the conventional natural gas conversion kit vehicles-computer controlled upstream injection. SAE paper 881659, 1988.

4 Huang, Q. and Chen, R. Prospects for a fluidic device based electronic gas fuel injection system for natural gas engines. In Proceedings of XXV FISITA Congress (3), on Automobile in Harmony with Human Society, 1994, paper 945128.

5 Huang, Q. and Chen, R. An investigation into the use of fluidic devices as gas fuel injectors for natural gas engines. SAE paper 960768, SP-1160, 1996.

6 Drzewiecki, T. M. and Goto, J. M. An analytical model for the response of fluidic wall attachment amplifiers. HDL paper TR-1598, June 1972.

7 Bourque, C. and Newman, B. G. Reattachment of a two dimensional incompressible jet to an adjacent flat plate, Aeronaut. Q., 1960, 11.

8 Bourque, C. Reattachment of a two-dimensional jet to an adjacent flat plate. In 1967 Fluidic Symposium on Advances in Fluidics, May 1967, pp. 192-204 (American Society of Mechanical Engineers, New York).

9 Foster, K. and Parker, G. A. Fluidics-Components and Circuits, 1970 (John Wiley-Interscience, London).

10 Kirshner, J. M. and Katz, S. Design Theory of Fluidic Components, 1975 (Academic Press, New York).

11 Epstein, M., Theoretical investigation of the switching mechanism in a bistable wall attachment fluid amplifier. Trans. ASME, J. Basic Engng, March 1971, 93, 55-62. 